\title{
Droit, éthique et déontologie
}

\section{Hans Stalder}

Prof. Dr méd., membre de la rédaction

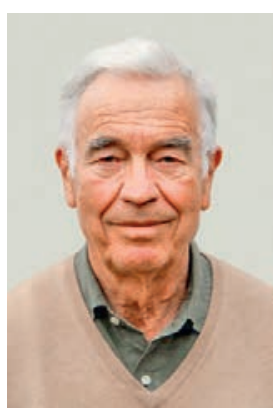

La publication des nouvelles directives de l'Académie suisse des sciences médicales (ASSM) «Attitude face à la fin de vie et à la mort» [1] a causé une réaction assez violente du Comité de la FMH et de la Société des médecins zurichois. Comment un avis éthique peut-il provoquer une opposition aussi forte? A mon avis, c'est parce la FMH a pris l'habitude d'appliquer les recommandations éthiques telles quelles dans son code de déontologie. Il faut cependant rappeler qu'il y a des différences entre l'éthique (qui réfléchit et pèse), la déontologie (qui donne des directives aux membres d'un corps professionnel) et le droit (qui autorise ou prohibe).

En ce qui concerne le suicide assisté, le droit suisse est clair et limpide: seul un motif égoïste est punissable; on peut en conclure que sans motif égoïste l'assistance est permise - aussi pour les médecins, puisque chacun est égal devant la loi.

Dans quelle situation l'assistance au suicide est éthiquement acceptable est une autre question. Le primum nil nocere doit être pesé entre l'autonomie du patient et la bienfaisance - par exemple le délivrer d'une souffrance inacceptable. Cela concerne toute personne qui prête son aide à un suicide et en particulier les médecins. Car ceux-ci sont principalement concernés, du fait qu'actuellement seuls les médecins sont autorisés à prescrire le pentobarbital et peuvent ainsi assister un malade dans son suicide d'une façon humaine.

Les textes de l'ASSM jouissent d'une bonne réputation. Ils ont cependant la particularité de n'être pas de simples prises de positions éthiques laissant aux médecins leur libre décision, mais d'être, comme l'indique leur dénomination, de véritables directives qui, au lieu de simplement peser le pour et le contre d'une action médicale difficile, donnent souvent des instructions précises. Et c'est ainsi que des positions éthiques présentées dans les directives sont reprises telles quelles dans le code déontologique de la FMH. La déontologie n'est cependant pas une réflexion éthique, même si elle s'en inspire, mais «l'ensemble des règles et des devoirs qui régissent une profession, la conduite de ceux qui l'exercent, les rapports entre ceux-ci et leurs clients et le public» (Larousse). Il s'agit donc de règles de comportement que la profession impose à ses membres, basées sur l'éthique et sur le droit. Mais elles peuvent aussi interdire aux professionnels ce que le droit par ailleurs permettrait.

Le fait qu'en plus les tribunaux se sont souvent basés sur les directives de l'ASSM pour arrêter leurs décisions rend la confusion d'autant plus grande.

Les nouvelles directives sont contestées par certains médecins parce qu'elles leur donneraient une responsabilité non désirée pour juger de la légitimité d'un suicide, qui pourrait être mise en cause par les tribunaux. Ils demandent une nouvelle loi qui règlerait définitivement ce problème de responsabilité en proposant une instance d'expertise composée de médecins indépendants, de juristes et de travailleurs sociaux [2]. Un tel comité serait un obstacle bureaucratique qui viendrait fortement compliquer des situations délicates où, il faut le souligner, le patient reste entièrement responsable de son acte, car c'est lui qui initie la procédure et décide de prendre le médicament mortel. Le médecin aide à réaliser, faute de mieux, ce qui est parfaitement permis par la loi. Et comme le montre l'exemple des associations d'aide au suicide, le médecin ne sera pas poursuivi, même s'il agit contre le code actuel de déontologie. C'est pourquoi la FMH devrait se garder de prohiber par le code de déontologie des actions qui sont permises par la loi et éthiquement acceptables. Soyons raisonnables: la population suisse veut avoir accès à l'assistance au suicide. Le médecin est le seul qui peut y contribuer humainement. Il le peut, mais ne le doit pas. Cette pratique s'est bien établie et fonctionne bien en Suisse. C'est pourquoi nous n'avons pas besoin d'une nouvelle loi.

\section{Références}

1 https://www.assm.ch/fr.html

2 https://www.aerzte-fuer-zuerich.ch/medien/2018-06-06-lockerung-der-aerztlichen-suizidbeihilfe-bei-nicht-toedlichenkrankheiten-braucht?_cldee=Ymtlc3NlbGlAZW1oLmNo\&recipientid=contact -6545 c5 cba69ce71180e-

93863bb351d40-f0346820a9924377ac83f525b1310338\&e sid $=6046013 a-456 b-e 811-8108-3863 b b 351 d 40 \& u r l i d=1$ 